\title{
Oridonin ameliorates depressive-like behaviors induced by chronic unpredictable mild stress in mice via TXNIP/NLRP3 signaling pathway
}

\author{
Lina Wang*, Ziye Huang, Jing Ping, XingYan Liu, Ziyao Cai, Chenghao Dai \\ Department of Psychiatry, Whenzhou No. 7 People's Hospital, Wenzhou City, Zhejiang Province 325000, China
}

*For correspondence: Email: linawang2082@163.com; Tel: +86-0577-89870026

Sent for review: 6 January 2021

Revised accepted: 8 March 2021

\begin{abstract}
Purpose: To investigate the effect and mechanism of oridonin in chronic unpredictable mild stress (CUMS)-induced depressive-like behaviors.

Methods: CUMS was established using 6-week stress stimuli, including feed/water deprivation, night lighting, inverted light/dark cycle, and tail clamping. Depressive behaviors were analyzed using the sucrose preference test, forced swim test (FST), and tail suspension test (TST). Locomotor activity was analyzed using the open field test (OFT) while inflammatory cytokines were analyzed by enzyme-linked immunosorbent assay. The activation of the TXNIP/NLRP3 signaling pathway was evaluated by western blot.

Results: Sucrose consumption of CUMS-treated mice was significantly decreased, while immobility times of the FST (control vs. CUMS, $\sim 50$ to $150 \mathrm{~s} ; p<0.01$ ) and TST (Control vs. CUMS, 50 to 130 s; $p<0.01)$ were increased; oridonin significantly reversed these effects. Spontaneous locomotor activities (crossing, rearing, and grooming) measured in the OFT were decreased after the CUMS procedure, and oridonin increased these activities ( $p<0.01$ vs. CUMS). Oridonin decreased the production of tumor necrosis factor alpha, interleukin (IL)-1 $\beta$, IL-6, and monocyte chemoattractant protein-1 in the hippocampus of CUMS-treated mice and significantly inhibited activation of the TXNIP/NLRP3 pathway induced by CUMS.

Conclusion: Oridonin ameliorates depressive-like behaviors in mice induced by CUMS, partly via TXNIP/NLRP3 signaling pathway. Thus, the findings provide evidence for the potential application of oridonin in depression therapy.
\end{abstract}

Keywords: Oridonin, Depression, Chronic unpredictable mild stress, TXNIP/NLRP3 signaling pathway

\begin{abstract}
This is an Open Access article that uses a fund-ing model which does not charge readers or their institutions for access and distributed under the terms of the Creative Commons Attribution License (http://creativecommons.org/licenses/by/4.0) and the Budapest Open Access Initiative (http://www.budapestopenaccessinitiative.org/read), which permit unrestricted use, distribution, and reproduction in any medium, provided the original work is properly credited.

Tropical Journal of Pharmaceutical Research is indexed by Science Citation Index (SciSearch), Scopus, International Pharmaceutical Abstract, Chemical Abstracts, Embase, Index Copernicus, EBSCO, African Index Medicus, JournalSeek, Journal Citation Reports/Science Edition, Directory of Open Access Journals (DOAJ), African Journal Online, Bioline International, Open-J-Gate and Pharmacy Abstracts
\end{abstract}

\section{INTRODUCTION}

Depression, a condition of mental disturbance, represents a series of presentations characterized by physical, emotional, and related cognitive disorders [1]. The World Health
Organization (WHO) has ranked depression as the fourth prime contributor of worldwide disease burden by 2020 [2]. Chronic stress is a primary factor of depression, the cause of which is immune disorders and inflammation [3]. Studies have demonstrated that inflammation is related 
to the complex process of depression [4]. Although many antidepressants improve depressive symptoms, they are associated with undesirable side effects, including suicidal tendencies, sexual dysfunction, and sleep disturbances [5]. Therefore, studies on antidepressants are urgent.

Thioredoxin-interacting protein (TXNIP), which detaches from thioredoxin (TRX) and binds to nucleotide-binding oligomerization domain-like receptor protein 3 (NLRP3), participates in inflammation-related diseases [6]. TXNIP is also a negative regulator of TRX and is considered a stress protein and key regulator of the inflammatory response. Increasing evidence has shown that the elevated expression of TXNIP is related to chronic stress and depression, and TXNIP deficiency inhibits inflammatory responses [7].

Oridonin (Figure 1A), a natural bioactive tetracycline diterpenoid, is a flavonoid compound isolated from Rabdosia rubescens that was first identified as an antitumor compound [8]. It has attracted increased attention because of its various pharmacological effects, including antiinflammatory and anti-oxidant activities [9]. For example, oridonin binds NLRP3, a central component of the inflammasome, and further inhibits NLRP3 inflammasome activation [10]. In vitro, oridonin inhibits the expression of inducible nitric oxide synthase and prostaglandinendoperoxide synthase 2 by inhibiting nuclear factor kappa B DNA binding activity [11]. Additionally, oridonin exerts a protective effect on the central nervous system. Zhang et al. found that oridonin significantly attenuated $\beta$-amyloid deposition and microglial activation in the mouse brain [12]. However, few studies have reported the effects of oridonin on depressive behaviors caused by chronic stress, and the relevant molecular mechanism requires further study. The present study aimed to investigate the effects of oridonin on chronic unpredictable mild stress (CUMS)-induced depressive-like behaviors and determine the mechanism.

\section{EXPERIMENTAL}

\section{Animals and treatment}

Twenty-four mice (C57BL/6J, approximately 6weeks old) were purchased from Beijing Vital River Laboratory Animal Technology. CUMS was established referring to a previously reported method s[3]. The stress stimuli lasted 6 weeks. The stress stimuli included feed/water deprivation $(24 \mathrm{~h})$, night lighting, tilting of the cage $\left(45^{\circ}\right)$, a wet cage $(200 \mathrm{~mL}$ of water/100 $\mathrm{g}$ of padding material), exposure to the external environment, an inverted light/dark cycle, tail clamping (1 $\mathrm{min})$, exposure in an empty bottle, strobe lighting, and shocking. The animals were divided into four groups: control, CUMS, CUMS + Oridonin (Ord, $10 \mathrm{mg} / \mathrm{kg}$ ), and CUMS + Fluoxetine (Flu, $10 \mathrm{mg} / \mathrm{kg}$ ). All the experiments were in accordance with the Guide for the Care and Use of Laboratory Animals [13] and were approved by the Ethics Committee of Whenzhou No. 7 People's Hospital (approval no. 2018117).

\section{Sucrose preference test}

The sucrose preference test (SPT) was performed on day 40. The animals had undergone adaptive training for $24 \mathrm{~h}$ with two bottles of $1 \%$ sucrose, and then one bottle was replaced with pure water for $24 \mathrm{~h}$. After $24 \mathrm{~h}$ of feed and water deprivation, each animal was given a bottle of pure water and $200 \mathrm{~mL}$ of another bottle of $1 \%$ sucrose solution. The consumption of pure water and sucrose was recorded after $1 \mathrm{~h}$ and again after $12 \mathrm{~h}$. To avoid the objective factors, the distance between the two bottles was kept unchanged and the positions were switched.

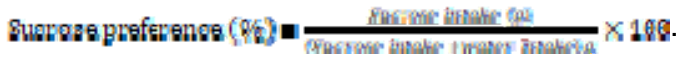

\section{Forced swimming test}

The forced swimming test (FST) apparatus was a cylinder filled with $10-\mathrm{cm}$-deep water $\left(23 \pm 2{ }^{\circ} \mathrm{C}\right)$. At the end of the CUMS protocol, a pretest was performed for $15 \mathrm{~min}$. For the pretest, the animals were allowed to swim individually for 15 min. After that, the animals were removed from the water, dried, and placed in a warm enclosure. After $24 \mathrm{~h}$, the procedure was repeated. The top view was recorded. Animals that floated without swimming were judged to be immobile. The immobility time was measured over 4 min after 6 min of adaptable swimming.

\section{Tail suspension test}

In the tail suspension test (TST), immobility was induced by suspending the mice by the tail. The animals were individually suspended from the tip of the tail $50 \mathrm{~cm}$ above the ground for 6 minutes. The mice remaining completely motionless were considered immobile.

\section{Open field test}

Locomotor activity was evaluated using the open field test (OFT). The apparatus was a black square cage with the floor divided into 12 equal squares. Animals freely explored the 
environment for $4 \mathrm{~min}$, with $30 \mathrm{sec}$ to adapt to the environment. The numbers of square crossing, grooming, and rearing events were recorded by a camera above the field.

\section{Determination of cytokines in the hippocampus}

The hippocampus of the mice was removed and homogenized in normal saline. The total protein was quantified using the bicinchoninic acid (BCA) method. The levels of tumor necrosis factor alpha (TNFa), interleukin (IL)-1 $\beta$, IL-6, and monocyte chemoattractant protein-1 (MCP-1) were analyzed by the enzyme-linked immunosorbent assay (ELISA).

\section{Western blotting}

Cells were lysed with RIPA lysis buffer, and total protein concentrations were quantified using the BCA method. Equal amounts of cell lysates were separated using SDS-PAGE and transferred onto polyvinylidene fluoride membranes. After blocking, each membrane was incubated with antibodies (1:1000 dilution) overnight at $4{ }^{\circ} \mathrm{C}$ and then incubated with the secondary antibody. The antibodies were as follows: anti-TXNIP (CST; \#14715), anti-NLRP3 (CST; \#13158), cleavedcaspase 1 (CST; \#89332), and HRP-linked antirabbit IgG (CST; \#7074). Finally, protein expression was visualized using an ECL system and analyzed by ImageJ software.

\section{Statistical analysis}

All the data were shown as means \pm standard deviation (SD) and analyzed using one-way analysis of variance (ANOVA) with Tukey's multiple comparison test as the post hoc test. $p<$ 0.05 was considered statistically significant.

\section{RESULTS}

\section{Effect of oridonin on the motivational behavior of CUMS mice}

The effects of oridonin (chemical structure shown in Figure $1 \mathrm{~A}$ ) on motivational behavior were analyzed using the SPT, TST, and FST. Anhedonia is a major characteristic of depression, measured in this study by reduced consumption of sucrose. The sucrose consumption of CUMS-treated mice was significantly decreased compared with that of the controls (Figure $1 \mathrm{~B} ; p<0.01$ ), and oridonin (Ord) and fluoxetine (Flu) treatment attenuated the decreasing consumption $(p<0.01$ vs. CUMS). The immobility time in the FST (Figure 1 C) and TST (Figure 1 D) was increased in CUMS mice ( $p<0.01$ vs. control), and oridonin and fluoxetine reduced the immobility time compared with mice treated with CUMS alone $(p<0.01)$. Fluoxetine treatment was more effective than oridonin treatment in motivational behavior improvement.

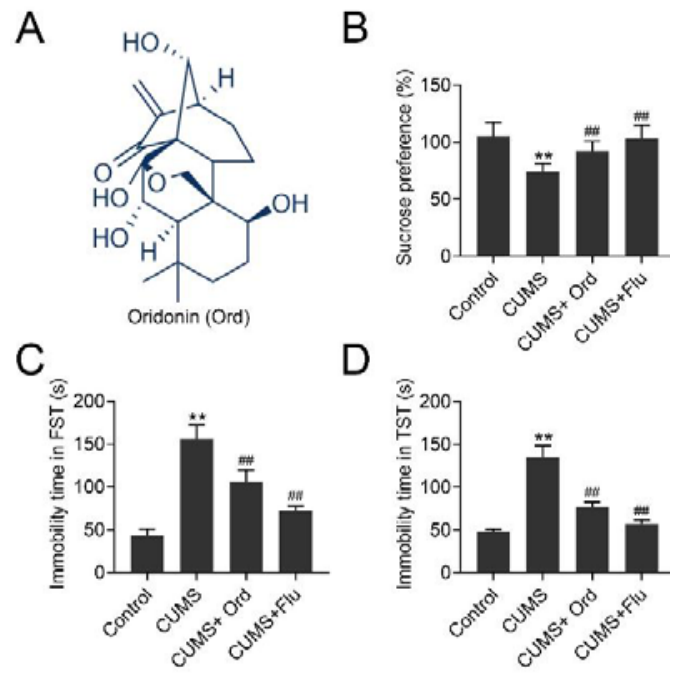

Figure 1: Effect of oridonin (Ord) and fluoxetine (Flu) on the motivational behavior of chronic unpredictable mild stress (CUMS) mice. (A) Chemical structure of oridonin. The sucrose preference (B), immobility time in the forced swimming test (FST) (C) and tail suspension test (TST) (D) of CUMS and Ord-treated mice; $\mathrm{n}=6,{ }^{* *} p<0.01$ vs. Control; ${ }^{\#} p<0.01$ vs. CUMS

\section{Oridonin affects the locomotor activity of CUMS-treated mice}

Spontaneous locomotor activities were measured in OFT. In the CUMS-treated group, the number of crossing, rearing, and grooming events decreased significantly ( $p<0.01$ vs. control). Oridonin and fluoxetine reversed the effects of CUMS treatment ( $p<0.01$ vs. CUMS), and the number of events in the fluoxetine group was closer to that of control (Figure 2).

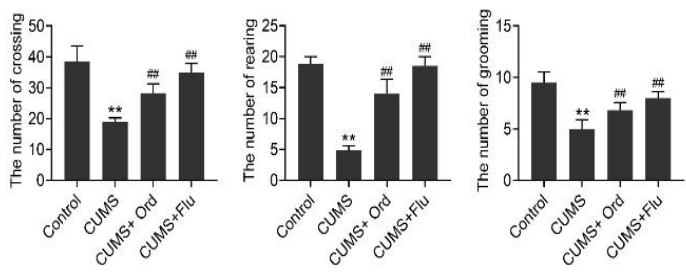

Figure 2: Oridonin (Ord) and fluoxetine (Flu) affect the locomotor activity of (chronic unpredictable mild stress) CUMS-treated mice. Number of crossing, rearing, and grooming events in the open field test; $\mathrm{n}=$ $6,{ }^{* *} p<0.01$ vs. Control; ${ }^{\# \#} p<0.01$ vs. CUMS 
Oridonin decreases the production of cytokines in the hippocampus

The levels of TNFa, IL-1 $1 \beta$, IL-6, and MCP-1 were dramatically increased in the CUMS group $(p<$ 0.01 vs. control), and oridonin and fluoxetine significantly inhibited the CUMS-induced increases of these cytokines in the hippocampus $(p<0.01$ vs. CUMS) (Figure 3$)$, indicating that oridonin attenuated the excess inflammatory responses induced by CUMS.
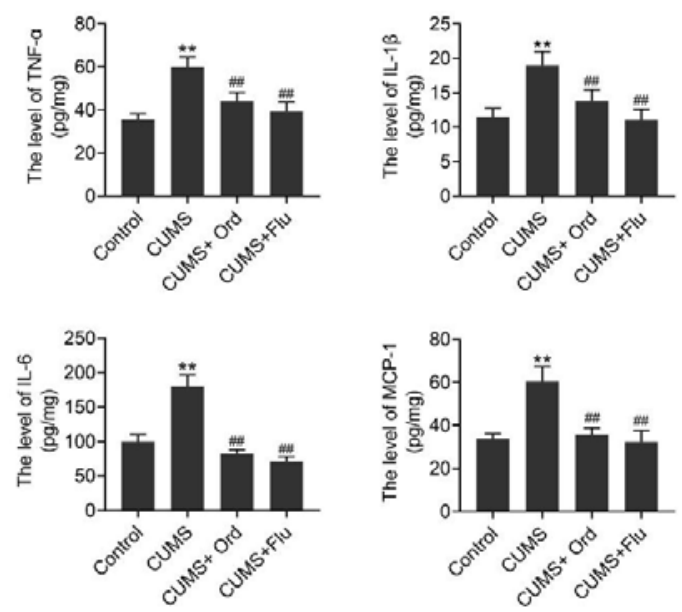

Figure 3: Oridonin (Ord) and fluoxetine (Flu) decrease the production of cytokines in the hippocampus. The levels of tumor necrosis factor alpha (TNFa), interleukin (IL)-1 $\beta$, IL-6, and monocyte chemoattractant protein-1 (MCP-1) in the hippocampus were analyzed by enzyme-linked immunosorbent assay; $\mathrm{n}=6,{ }^{* *} p<0.01$ vs. Control; \#\# $p<0.01$ vs. CUMS

Effect of oridonin on the expression of TXNIP/NLRP3 pathway-related proteins

To investigate the effects of oridonin on inflammatory responses, TXNIP/NLRP3 pathway-related proteins were analyzed by western blot. We previously showed that, in CUMS-treated mice, TXNIP/NLRP3 is activated, and the expression of TXNIP, NLRP3, and cleaved caspase-1 increased (Figure 4). As expected, oridonin treatment significantly decreased the levels of TXNIP/NLRP3 proteins $(p<0.01$ vs. CUMS).

\section{DISCUSSION}

Chronic stress is a major risk factor for depression, a chronic and recurrent syndrome of mood disorder. CUMS is a model that mimics stress-induced depression and has been widely used in preclinical research [3].
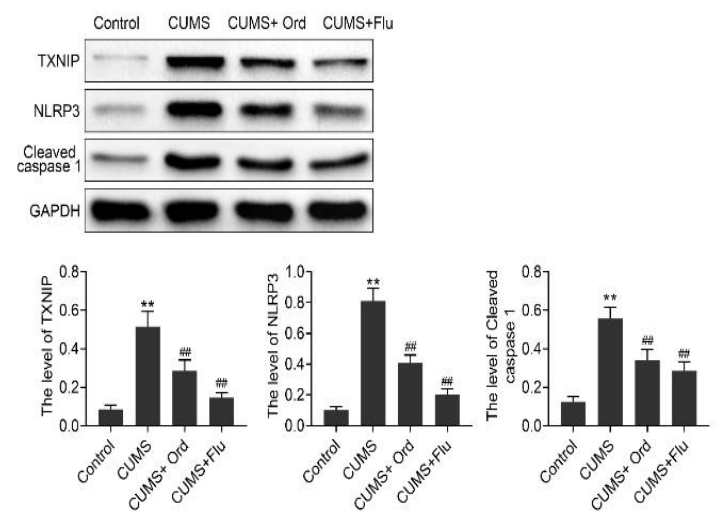

Figure 4: Effect of oridonin (Ord) and fluoxetine (Flu) on the expression of TXNIP/NLRP3 pathway-related proteins. Expression of thioredoxin-interacting protein (TXNIP), nucleotide-binding oligomerization domainlike receptor protein 3 (NLRP3), and cleaved caspase 1 in the hippocampus was analyzed by western blot; ${ }^{* *} p<0.01$ vs. Control; ${ }^{\# \#} p<0.01$ vs. CUMS

Previous studies have confirmed that the CUMS model shows depressive behavior, such as decreased sucrose consumption [14]. In the present study, after the CUMS procedure (45 days), mice displayed depressive-like behaviors and cognitive defects, including less sucrose consumption, increased immobility time, and a reduced number of crossing, rearing, and grooming events in the OFT. However, oridonin administration reversed these depressive-like behaviors in CUMS mice, similar to the positive drug (fluoxetine).

Depression is associated with inflammatory responses [15], and depressed patients demonstrate abnormal production of proinflammatory cytokines [4]. Studies have also shown that pro-inflammation cytokines play a key role in the progression of inflammation, which induces depressive behaviors [16]. Many studies have shown that attenuating inflammation in the hippocampus significantly alleviates depressive behaviors [17]. For example, baicalein, a flavonoid glycoside isolated from Radix Scutellariae, ameliorates neuro-inflammation and depressive behaviors by inhibiting the phosphoinositide 3-kinase/AKT pathway [18]. Oridonin has garnered increased attention because of its extensive biological activities, including anti-inflammatory effects. $\mathrm{Xu}$ et al. reviewed the effects of oridonin and its analogs on inflammation and highlighted its role as a therapeutic candidate in neuroprotection [19]. In the present study, the levels of pro-inflammation cytokines in the hippocampus were increased significantly after CUMS treatment and were all inhibited by oridonin, suggesting a potential antiinflammatory effect of oridonin in depression. 
TXNIP, an inhibitor of TRX, is now known to be a critical regulator of NLRP3 inflammasome activation [20]. TXNIP overexpression activates inflammatory pathways via TRX-mediated inflammation inhibition or by directly activating the NLRP3 inflammasome [21]. Previous studies have suggested that TXNIP dissociates from TRX and binds to NLRP3, a multiple protein complex comprising NLRP3, apoptosisassociated speck-like protein, and caspase-1. The assembly of this complex results in caspase1 activation, further producing mature IL-1 $\beta$, which plays a central role in inflammation [22]. Thus, in the present study, activation of the TXNIP/NLRP3 signaling pathway was analyzed. Oridonin inhibited the expression of TXNIP, NLRP3, and cleaved caspase-1 induced by CUMS, suggesting a possible mechanism for the anti-depression effects of oridonin. A similar effect of oridonin was found in mice with myocardial ischemia/reperfusion (I/R) injury [10]. Oridonin alleviated I/R induced myocardial injury by inhibiting oxidative stress and the NLRP3 inflammasome pathway [10].

\section{CONCLUSION}

The findings of this study suggest that oridonin ameliorates depressive-like behaviors of CUMS mice partly via TXNIP/NLRP3 signaling pathway, and thus, provide evidence for the potential application of oridonin in depression therapy.

\section{DECLARATIONS}

\section{Conflict of interest}

No conflict of interest is associated with this work.

\section{Contribution of authors}

We declare that this work was performed by the authors named in this article and all liabilities pertaining to claims relating to the content of this article will be borne by the authors. Lina Wang and Ziye Huang designed the study and supervised the data collection. Jing Ping analyzed and interpreted the data. XingYan Liu, Ziyao Cai, and Chenghao Dai prepared the manuscript for publication and reviewed the draft of the manuscript. All the authors read and approved the manuscript.

\section{Open Access}

This is an Open Access article that uses a funding model which does not charge readers or their institutions for access and distributed under the terms of the Creative Commons Attribution License (http://creativecommons.org/licenses/by/ 4.0) and the Budapest Open Access Initiative (http://www.budapestopenaccessinitiative.org/rea d), which permit unrestricted use, distribution, and reproduction in any medium, provided the original work is properly credited.

\section{REFERENCES}

1. Smith K. Mental health: a world of depression. Nature 2014; 515(7526): 181.

2. Colledge S, Larney S, Peacock A, Leung J, Hickman M, Grebely J, Farrell M, Degenhardt L. Depression, posttraumatic stress disorder, suicidality and self-harm among people who inject drugs: A systematic review and meta-analysis. Drug Alcohol Depend 2020; 207: 107793.

3. Antoniuk S, Bijata M, Ponimaskin E, Wlodarczyk J. Chronic unpredictable mild stress for modeling depression in rodents: Meta-analysis of model reliability. Neurosci Biobehav Rev 2019; 99: 01-116.

4. Dowlati $Y$, Herrmann $N$, Swardfager $W$, Liu H, Sham L, Reim EK, Lanctôt KL. A meta-analysis of cytokines in major depression. Biol Psychiatry 2010; 67(5): 446-457.

5. Zhang $L$, Long $M, X u L$. Comparative studies on the therapeutic and adverse effects of mirtazapine and fluoxetine in the treatment of adult depression. Trop $J$ Pharm Res 2019; 18(1).

6. Nasoohi S, Ismael S, Ishrat T. Thioredoxin-Interacting Protein (TXNIP) in Cerebrovascular and Neurodegenerative Diseases: Regulation and Implication. Mol Neurobiol 2018; 55(10): 7900-7920.

7. Song Y, Sun R, Ji Z, Li X, Fu Q, Ma S. Perilla aldehyde attenuates CUMS-induced depressive-like behaviors via regulating TXNIP/TRX/NLRP3 pathway in rats. Life Sci 2018; 206: 117-124.

8. Li D, Han T, Liao J, Hu X, Xu S, Tian K, Gu X, Cheng K, $\mathrm{Li} Z$, Hua $H$ et al. Oridonin, a Promising ent-Kaurane Diterpenoid Lead Compound. Int J Mol Sci 2016; 17(9): 1395.

9. Cheng W, Huang C, Ma W, Tian X, Zhang X. Recent Development of Oridonin Derivatives with Diverse Pharmacological Activities. Mini Rev Med Chem 2019; 19(2): 114-124.

10. Lu C, Chen C, Chen A, Wu Y, Wen J, Huang F, Zeng Z. Oridonin Attenuates Myocardial Ischemia/Reperfusion Injury via Downregulating Oxidative Stress and NLRP3 Inflammasome Pathway in Mice. Evidence-based complementary and alternative medicine: eCAM 2020; 2020: 7395187-7395187.

11. Zhao G, Zhang T, Ma $X$, Jiang $K, W u H$, Qiu C, Guo M, Deng G. Oridonin attenuates the release of proinflammatory cytokines in lipopolysaccharide-induced RAW264.7 cells and acute lung injury. Oncotarget 2017; 8(40): 68153-68164.

Trop J Pharm Res, March 2021; 20(3): 535 
12. Zhang Z-Y, Daniels R, Schluesener HJ. Oridonin ameliorates neuropathological changes and behavioural deficits in a mouse model of cerebral amyloidosis. Journal of cellular and molecular medicine 2013; 17(12): 1566-1576.

13. Dolgun Z, Altintas A, Ivan C, Balkanli P. Comparison of preoperative magnetic resonance imaging results with postoperative pathologic results in early-stage uterine cervical cancer. Eur J Gynaecol Oncol 2018; 39(6): 935938.

14. Fu H, Liu L, Tong Y, Li Y, Zhang X, Gao X, Yong J, Zhao $J$, Xiao $D$, Wen $K$ et al. The antidepressant effects of hesperidin on chronic unpredictable mild stress-induced mice. Eur J Pharmacol 2019; 853: 236-246.

15. Kohler O, Krogh J, Mors O, Benros ME. Inflammation in Depression and the Potential for Anti-Inflammatory Treatment. Curr Neuropharmacol 2016; 14(7): 732-742.

16. Ng A, Tam WW, Zhang MW, Ho CS, Husain SF, McIntyre RS, Ho RC. IL-1 $\beta$, IL-6, TNF- $\alpha$ and CRP in Elderly Patients with Depression or Alzheimer's disease: Systematic Review and Meta-Analysis. Sci Rep 2018; 8(1): 12050.
17. Lang UE, Borgwardt S. Molecular mechanisms of depression: perspectives on new treatment strategies. Cell Physiol Biochem 2013; 31(6): 761-777.

18. Guo L-T, Wang S-Q, Su J, Xu L-X, Ji Z-Y, Zhang R-Y, Zhao $Q-W$, Ma Z-Q, Deng $X-Y$, Ma S-P. Baicalin ameliorates neuroinflammation-induced depressive-like behavior through inhibition of toll-like receptor 4 expression via the PI3K/AKT/FoxO1 pathway. J Neuroinflamm 2019; 16(1): 95-95.

19. Xu J, Wold EA, Ding Y, Shen Q, Zhou J. Therapeutic Potential of Oridonin and Its Analogs: From Anticancer and Antiinflammation to Neuroprotection. Molecules (Basel, Switzerland) 2018; 23(2): 474.

20. Yang C, Xia W, Liu X, Lin J, Wu A. Role of TXNIP/NLRP3 in sepsis-induced myocardial dysfunction. Int J Mol Med 2019; 44(2): 417-426.

21. Wen Y, Liu YR, Tang TT, Pan MM, Xu SC, Ma KL, Lv LL, Liu H, Liu BC. mROS-TXNIP axis activates NLRP3 inflammasome to mediate renal injury during ischemic AKI. Int J Biochem Cell Biol 2018; 98: 43-53.

22. Yue $L M$, Gao YM, Han BH. Evaluation on the effect of hydrogen sulfide on the NLRP3 signaling pathway and its involvement in the pathogenesis of atherosclerosis. $J$ Cell Biochem 2019; 120(1): 481-492. 